\title{
Marco general de análisis de la formación logística en Colombia*
}

DOI: http://dx.doi.org/10.21830/19006586.80

Recibido: 29 de agosto de 2016 - Aceptado: 2 de noviembre de 2016

General framework of analysis of logistic training in Colombia

Cadre général d’analyse de la formation logistique en Colombie

Quadro geral da análise da formação logística na Colômbia

Milton Ricardo Ospina Díaz
Pedro Emilio Sanabria Rangel

* Artículo derivado del trabajo de la línea de investigación en Estudios Contemporáneos en Gestión y Organizaciones (sublínea en Estrategia, Innovación y Competitividad) del Grupo de Estudios Contemporáneos en Contabilidad, Gestión y Organizaciones de la Universidad Militar Nueva Granada.

a Docente de tiempo completo de la Facultad de Ciencias Económicas de la Universidad Militar Nueva Granada (Bogotá). Administrador de Empresas de la Universidad Militar Nueva Granada. Especialista en Mercadeo de Servicios de la Universidad Militar Nueva Granada. Especialista en Pedagogía de la Universidad La Gran Colombia. Especialista en Logística de la Escuela Logística del Ejército. Magíster en Gestión de Organizaciones de la Universidad Militar Nueva Granada. Profesor de posgrado y pregrado en el área de Mercadeo en varias universidades en Colombia. Consultor en Mercadeo. Exdecano de la Escuela Logística del Ejército. <milton.ospina@unimilitar. edu.co>; <milton_ospinad@hotmail.com>

b Docente de tiempo completo de la Facultad de Ciencias Económicas de la Universidad Militar Nueva Granada (Bogotá). Administrador de Empresas y Magíster (M.Sc.) en Administración de la Universidad Nacional de Colombia; Diplôme d'université "Master II" Sciences de Gestion de la Universidad de Rouen (Francia) y Doctorado (c) en Bioética de la Universidad Militar Nueva Granada . Investigador adscrito al Grupo de Estudios Contemporáneos en Contabilidad, Gestión y Organizaciones de la Universidad Militar Nueva Granada. Docente Ocasional de posgrado en varias universidades en Colombia. Exdirector de Posgrados y Excoordinador de la Maestría en Gestión de Organizaciones de la Facultad de Ciencias Económicas de la Universidad Militar Nueva Granada.<pedro.sanabria@unimilitar.edu.co>; <pesanabriar@gmail.com>. Identificador ORCID: https://orcid. org/0000-0001-7018-9417 
Resumen. Aquí se pretende realizar una revisión sobre la logística en Colombia que permita caracterizar su historia y desarrollo, entender su contexto actual y comprender la demanda formativa generada. También se hace un análisis de la oferta logística existente en el país en los diferentes niveles de formación. Se estudia la correspondencia entre los requerimientos sociales y las condiciones de la oferta académica en el campo. Con este fin se hace una revisión de la literatura internacional sobre el tema, para entender el estado global del campo que sirve de base para la discusión, y de la literatura nacional, con el fin de comprender la situación y contexto de Colombia, especialmente en el marco del posconflicto. Se revisaron documentos de organismos nacionales e internacionales, relacionados con el campo logístico, que alimentan la discusión al respecto. Posteriormente se realizaron síntesis y comparativos que permitieron construir el marco general para el estudio. Basado en ellos, se llevó a cabo una caracterización general de la logística, con énfasis en el caso colombiano, y un análisis del estado actual de la formación logística en el país. En general se encuentra que existe un sinnúmero de estudios nacionales e internacionales sobre el tema logístico, lo cual evidencia una preocupación creciente sobre este, y que tal interés se ha visto acompañado, en Colombia, por un crecimiento en el número de programas académicos en el campo, en los diferentes niveles de formación. No obstante, existen aspectos por revisar para las diferentes regiones y nuevos retos para la oferta formativa.

Palabras clave: historia de la logística; logística militar; logística colombiana; formación logística; educación en logística.

Abstract. The present work intends to make review on the state of the art of logistics in Colombia that allows to characterize its history and development, understand its current context and understand the demand for training in this area of knowledge. The present work also analyzes the current educational offer in logistics in Colombia at the different levels of formation. The coherence between the social requirements and the current conditions of the academic offer in logistics is studied. For this purpose, a global review of the literature is made in order to understand the state of the art of logistics worldwide, which will be the basis for the discussion; and a local review is also made in order to understand the situation and context of Colombia, especially within the context of the post-conflict. Logistics-related documents of Colombian and international organizations were reviewed to feed the discussion. Afterwards, synthesis and comparisons were made to allow the construction of the theoretical framework for this study. Based on these synthesis and comparisons a general characterization of logistics was made, emphasizing on Colombia and the current situation of the academic offer in logistics. In general terms it was found that there is a large number of local and international studies on logistics, which is an evidence of the growing concern about this area, and in Colombia this interest in logistics is parallel to the increase in the number of academic programs in this area at different levels of formation. However, there are still issues to review in the different regions and new challenges for the upcoming academic offers.

Keywords: logistics history; military logistics; Colombian logistics; logistics training; logistics education.

Résumé. Cette étude analyse la logistique en Colombie pour caractériser son histoire et évolution, comprendre son contexte actuel et comprendre la demande d'éducation dans ce domaine. Il y aborde aussi l'offre académique en logistique en Colombie à différents niveaux de formation. On y examine la correspondance entre les exigences sociales et les conditions de l'offre académique existante. À cette fin, on évalue la littérature au niveau international pour comprendre la situation de la logistique en Colombie, en particulier dans le contexte d'après conflit. On y analyse documents d'organisations nationales et internationales connexes liés à la logistique pour alimenter la discussion. De plus, une synthèse comparative est effectuée pour construire le cadre théorique. Sur la base de la synthèse comparative une caractérisation générale de la logistique en Colombie et une analyse de l'état actuel de la formation en logistique en Colombie sont effectuées. D'une manière générale, l'on a trouvé qu'il existe un très grand nombre d' 
études nationales et internationales sur la logistique, en soulignant qu'il y a un grande préoccupation liée à la logistique, et que cet intérêt est accompagné, en Colombie, d'un nombre croissant de programmes d'enseignement à différents niveaux de formation. Cependant, il y a aspects pour observer dans les différents régions et nouveaux défis pour les futurs programmes d'enseignement en logistique.

Mots-clés: histoire logistique; logistique militaire; logistique colombienne; formation logistique; éducation en logistique.

Resumo. Este estudo faz uma análise da logística na Colômbia para caracterizar sua história e desenvolvimento, compreender seu contexto atual e entender a demanda de treinamento nesta área. Faz-se também uma análise das opções acadêmicas existentes em logística na Colômbia em diferentes níveis de formação. Estuda-se a correspondência entre os requisitos sociais e as condiçóes da oferta acadêmica existente. Para este fim, faz-se tanto uma revisão da literatura sobre o assunto a nível internacional para compreender o estado geral da logística, que serve como base para a discussáo; quanto uma revisão a nível nacional para compreender a situação da logística na Colômbia, especialmente no contexto do pós-conflito. Analisaramse documentos de organizaçóes nacionais e internacionais relacionados com logística para basear a discussão. Alem disso, realizou-se uma síntese comparativa que permitiu a construção do quadro teórico para o estudo. Com base nela realizou-se uma caracterização geral da logística, com destaque para o caso colombiano, e uma análise do estado atual da formação em logística na Colômbia. Em geral, verificou-se que há uma série de estudos nacionais e internacionais sobre a questão logística, destacando uma crescente preocupação em relação à logística, e que esse interesse tem sido acompanhado, na Colômbia, pelo crescimento do número de programas acadêmicos nesta área, em diferentes níveis de formação. No entanto, existem aspectos para verificar nas diferentes regiôes e novos desafios para as futuras ofertas de formação.

Palavras chave: história logística; logística militar; logística da Colômbia; formação logística; educação em logística.

\section{Introducción}

Aunque la logística parece haber acompañado al hombre casi desde sus orígenes como especie, esta actividad se ha estado configurando paulatinamente como un campo de estudio completo, sobre todo si se considera la importancia que esta ha adquirido para las organizaciones en el desarrollo de su propósito. En tal sentido, si se considera que las organizaciones se han constituido en uno de los elementos más importantes en la estructura económica actual, es posible entender la creciente demanda social por talento humano con condiciones y competencias en el campo logístico que le permita desarrollar y mejorar la ejecución de la amplia gama de actividades que esta implica.

No obstante, cuando se revisan los estudios efectuados en el campo logístico no se encuentran análisis en relación con las condiciones y el número de los programas de formación existentes, que muestren la relación de la oferta de formación con la demanda social. Esto se hace más evidente cuando se revisa el caso de Colombia. De hecho, para el caso colombiano tampoco se encuentran referentes de análisis sobre el campo logístico que permitan caracterizar su desarrollo y entender el contexto actual en que se desenvuelve. Por ello, no se cuenta tampoco con un medio que haga posible comprender mejor la demanda existente en el país y el número de programas educativos requeridos en los diferentes niveles de formación. 
En este artículo se pretende entonces sistematizar la información disponible con respecto al campo logístico, especialmente para el caso colombiano, de forma que se cuente con una caracterización amplia de su estado actual. Esto puede dar luces de los requerimientos sociales que pueden existir en relación con expertos en este campo. Una vez hecho esto se realiza un análisis de los programas académicos en logística, con base en la oferta nacional, en los distintos niveles de formación, que permite determinar las condiciones actuales de la oferta académica.

Un análisis de este tipo es de utilidad para el campo logístico y las diferentes organizaciones que se sustentan en él, pues les permite entender el contexto actual de desarrollo y obtener en el futuro el talento humano adecuado, en número y competencias, frente a sus requerimientos. Asimismo, puede ser pertinente para el sector educativo y las diferentes instituciones académicas en función de determinar el estado actual de la formación en logística; esto puede constituirse en un referente que permita la toma de decisiones sobre la cantidad de programas a ofrecer en las diferentes regiones (regulación de la oferta) y los niveles en los cuales esto podría hacerse.

Según lo anterior, a continuación se hace una breve conceptualización sobre la logística y un recorrido por su desarrollo histórico; posteriormente, se hace una revisión de este concepto y una contextualización del caso colombiano para, finalmente, realizar un análisis de la oferta de formación en el campo logístico en Colombia.

\section{Conceptualización de la logística}

Como ocurre en muchas de las profesiones, se suele afirmar que la logística ha estado presente a lo largo de la historia, pues ha acompañado al ser humano en todas las actividades que han implicado algún nivel de organización del trabajo, aunque en la actualidad parece haber adquirido la mayor importancia en razón de los requerimientos e imaginarios que se le han atribuido a las organizaciones, especialmente a las empresariales.

Por tanto, la logística se considera una actividad fundamental en todas las organizaciones, pues tiene como propósito gestionar eficientemente el "conjunto de medios y métodos necesarios para llevar a cabo la organización de una empresa, o de un servicio, especialmente de distribución" (Real Academia Española - RAE, 2001). Es fundamental para el comercio y los negocios, ya que constituye un medio de enlace entre el sistema productivo y el mercado, cuando normalmente estos están separados por el tiempo y la distancia (Cedillo Campos \& Sánchez Ramírez, 2008).

Según Posada Zamudio (1997), la palabra logística proviene de la raíz griega logis, que significa 'cálculo', y del latín logisticus, que identificaba al intendente o administrador de los ejércitos del imperio romano. No obstante, según Puentes Garzón (2006) "etimológicamente, la palabra logística proviene del griego logistikós, que significa 'saber calcular' [...] [aunque][...] los romanos usaban la palabra logística al referirse a sus oficiales de ejércitos" (p. 35). Desde otra perspectiva se afirma que logística "es una palabra compuesta de los vocablos derivados del inglés logistics y del francés logistique y loger" (López Torres, Alcalá Álvarez, Plascencia López, \& Marín Vargas, 2010, p. 78).

De los orígenes etimológicos ya señalados se establece que la logística guarda una estrecha relación con el ámbito militar y que se considera parte esencial de este, pues es la que permite atender las necesidades y operaciones de los ejércitos en momentos de paz o en tiempos de guerra. Así, 
los asuntos logísticos "son los aspectos de las operaciones militares que tienen que ver con el diseño y desarrollo, adquisición, bodegaje, movimiento, distribución, mantenimiento, evacuación y disposición del material" (LOGMARS- Departamento de Defensa, apud Legiscomex, 2015).

No obstante, de manera más general, se puede entender también la logística como "un conjunto amplio de actividades relacionadas con el movimiento y almacenamiento de materiales, productos e información" (Velásquez Contreras, 2003, p. 69). Por su parte, el Consejo de Dirección Logística (CLM), citado en (Ballou, 2004), entiende la logística como "la parte del proceso de la cadena de suministros que planea, lleva a cabo y controla el flujo y almacenamiento eficientes y efectivos de bienes y servicios, así como de la información relacionada, desde el punto de origen hasta el punto de consumo, con el fin de satisfacer los requerimientos de los clientes" (p. 4).

De forma aún más amplia, Cipoletta, Pérez y Sánchez (2010) consideran la logística como "aquella parte de la Gestión de la Cadena de Suministros que planifica, implementa y controla el flujo — hacia atrás y hacia adelante — y el almacenamiento eficaz y eficiente de los bienes, servicios e información relacionada desde el punto de origen al punto de consumo, con el objetivo de satisfacer los requerimientos de los consumidores" (p. 16).

Adicionalmente, aunque de forma complementaria, también se considera la logística como el "proceso de planificación, ejecución y control de procedimientos para el transporte eficiente y eficaz y para el almacenamiento de bienes, incluyendo servicios e información relacionada, desde el punto de origen al punto de consumo con el fin de ajustarse a las necesidades del cliente. Esta definición incluye entradas, salidas y movimientos internos y externos" (Council of Supply Chain Management Professionals - CSCMP, 2013, p. 117).

Asimismo, se considera que la logística es

la disciplina responsable de administrar los flujos de bienes, servicios e información tanto al interior de las organizaciones, como a lo largo de las cadenas de suministro - CS_- compuestas por proveedores de bienes y servicios, fabricantes, distribuidores comerciales y clientes, para satisfacer la demanda de estos últimos, generar rentabilidad y sostenibilidad para las organizaciones integrantes de la cadena y aportar bienestar a la sociedad (Closs \& Stank, 1999, apud Mendoza, 2013, p. 58).

Así, el concepto de logística incluye la gestión, planificación y administración de las actividades que forman parte en las diversas funciones, áreas, procesos y sistemas de las organizaciones: compras, producción, almacenaje, manutención, transporte y distribución.

En el ámbito empresarial existen múltiples definiciones del término logística, que ha evolucionado desde la logística militar hasta el concepto contemporáneo, que la muestra como una ciencia, disciplina, arte y técnica que se ocupa de la administración, organización y mantenimiento de los flujos de materiales, mercancías (bienes y servicios), energía, información y residuos en una organización hacia y desde el punto de consumo, con el fin de satisfacer las necesidades de las partes interesadas y aportar al bienestar social.

En la actualidad, el campo de la logística se ha constituido en un referente importante para la perdurabilidad y el desempeño de las organizaciones, pues en el marco del mercado y la globalización, se empieza a requerir que estas tengan fortaleza en la planeación logística; en las operaciones

1 Traducción de los autores. 
de almacenamiento, transporte y administración de centros de distribución; en el manejo de la cadena de suministro, y en el servicio al cliente.

De esta forma, en un mundo cada vez más dinámico, las organizaciones se ven enfrentadas a nuevos retos asociados a la innovación, al adecuado manejo del tiempo, a la producción flexible, al manejo adecuado de los niveles de productividad, al adecuado almacenamiento, a la eficiencia en el movimiento de bienes desde el punto de compra de materias primas e insumos hasta el punto de venta, y al adecuado manejo de los diferentes flujos de información generados en el proceso que les permitan brindarles a los consumidores un nivel de servicio adecuado y a un costo razonable (Ballou, 1999).

Como se puede observar, el concepto de logística se encuentra asociado también con nociones como las de gestión estratégica, la gestión de la cadena de suministro (Supply Chain Management - SCM-) y la logística inversa. Con todo esto se hace evidente el valor y papel estratégico que mantiene la logística.

La primera noción (gestión estratégica) se vincula con esta debido a que considera la logística como un medio que apoya la estrategia de las organizaciones mediante el estudio, administración y gestión integral de las actividades que permiten el seguimiento de las reglas y políticas establecidas, así como el cumplimiento de los objetivos organizacionales (Narasimhan, McLeavey, \& Billington, 1996, p. 28).

La segunda noción, es decir, la de gestión de la cadena de suministro, se relaciona con la logística porque esta última se encuentra referida al acto de planificación, implementación y control del flujo de materiales, información y productos dentro de la cadena que está entre la producción y el consumo final, y que permite la satisfacción de las necesidades de los consumidores (Lambert, Cooper, \& Pagh, 1998).

La tercera, la logística inversa, está relacionada en cuanto se encarga de determinar los procesos referidos al retorno de productos desde el consumidor hacia el productor, pasando por el reciclaje, la reutilización de materiales, la reutilización de componentes, la eliminación de residuos y las operaciones de restauración, reparación y refabricación (Schmidheiny, 1992). No obstante, este concepto no se usa solo para referirse al papel de la logística en el retorno de los productos, sino que está referido también a la reducción en origen, al reciclado, a la reutilización de materiales, a la sustitución de materiales, a la eliminación de residuos y desperdicios, a la reparación y a la remanufacturación de los productos — gestión ambiental— (Klaus, 1999).

Según las definiciones anteriores, en la figura 1 se compilan los aspectos más representativos. Allí se muestra la logística en sus tres fases: compras, producción y distribución física, mientras, a su vez, se evidencian las operaciones logísticas intervinientes en el ciclo logístico: planeación de la demanda, gestión de inventarios, transporte, almacenamiento, transformación, packing, picking y despacho de mercancía. 


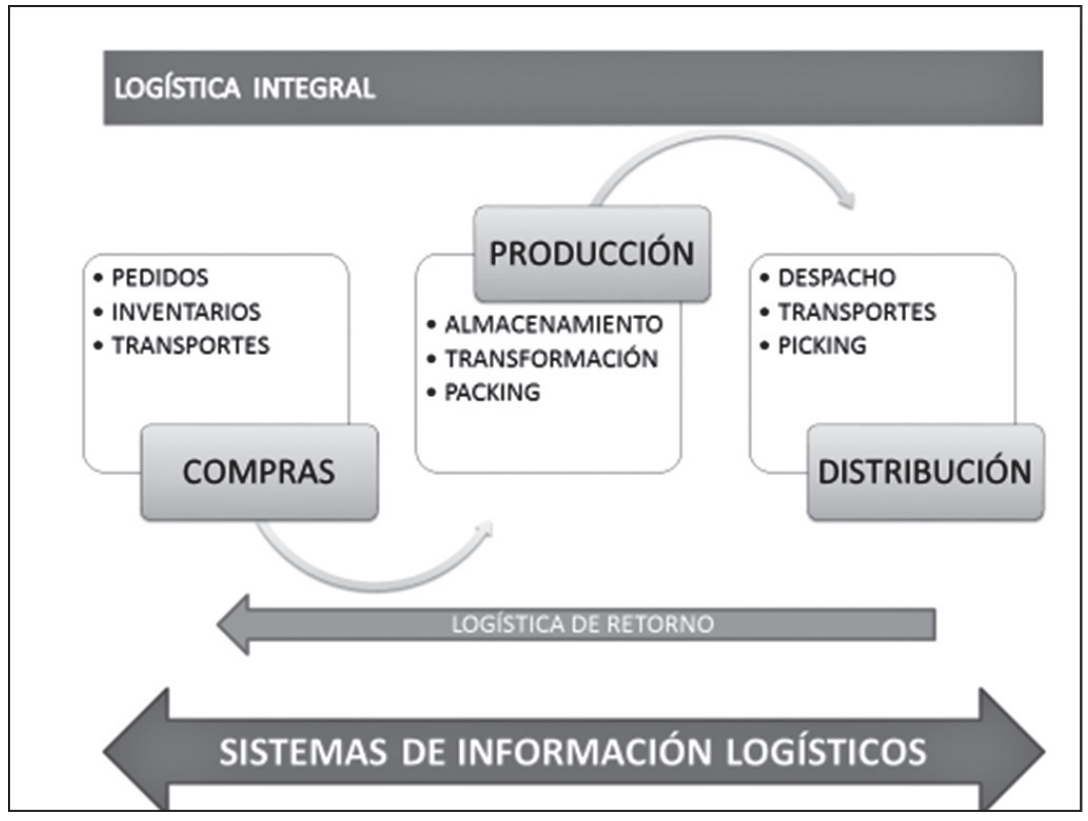

Figura 1. Proceso logístico integral y sus actividades Fuente: Elaborado por los autores

En esta gráfica se hace explícito el objetivo primordial de la logística, a saber: la optimización de los flujos de productos o servicios e información asociada, desde la planeación de necesidades referidas al abastecimiento de materia prima con los proveedores, hasta la entrega de productos o servicios al cliente final, incluyendo también la logística de retorno. Todo ello en el marco de criterios de eficiencia, de eficacia y de costo razonable que el modelo económico vigente ha impuesto para las organizaciones.

\section{Desarrollo histórico del concepto de logística}

Como ya se señaló, la logística parece haber estado presente a lo largo de la historia, puesto que el ser humano siempre ha requerido movilizar objetos de un lugar a otro y se ha enfrentado a la necesidad de establecer las mejores maneras para poder hacerlo adecuadamente y de forma ágil. No obstante, el concepto de logística encuentra en el contexto militar su referente más importante, si se quiere establecer un origen específico de la logística, de la forma como es entendida actualmente.

Sin embargo, en gran parte por el paso de la era industrial a la era de la información, el campo de aplicación de la logística ya no se encuentra circunscrito solamente al ámbito militar, sino que ha trascendido hacia diferentes campos, incluido el organizacional. En esta parte se muestra, de forma general, el proceso de evolución que esta ha tenido en función de la aplicación al campo militar y al ámbito empresarial (organizaciones). 


\section{Contexto militar}

La logística encuentra sus inicios en la historia de la guerra misma, es decir, en la historia de la humanidad. Esta disciplina ha evolucionado con el ser humano hasta convertirse en un aspecto fundamental para los procesos de apoyo a las unidades de combate. En general, la logística militar ha sido definida como "el componente del arte de la guerra que tiene por objeto proporcionar a los ejércitos los suministros necesarios para afrontar las exigencias de la guerra" (Posada Zamudio, 1997).

La logística militar ha sido desarrollada para atender las necesidades de los ejércitos en las operaciones y lograr el bienestar de la tropa en términos de alimentación, dotaciones, sanidad, armamento y transportes, con el fin de brindar el apoyo necesario de forma que pueda hacer frente a las limitaciones o retos que le imponen el clima, el enemigo y la geografía, entre otros aspectos, para dar cumplimiento a la misión que le es encomendada.

Este aspecto es tan importante para los ejércitos que se puede afirmar que la eficiencia en el manejo logístico ha determinado la derrota o la victoria en muchas batallas, puesto que sin suministros, ninguna tropa tiene posibilidades de desempeñarse adecuadamente en la guerra.

A raíz de este fenómeno se iniciaron los procesos de formación logística en las diferentes escuelas militares del mundo, pues se considera que un aprendizaje correcto del tema permite también un adecuado apoyo a las unidades de combate. Así, quienes están llamados a desempeñarse en cargos logísticos de unidades militares deben aprender a planear, organizar y dirigir el flujo de material de intendencia, el armamento, las municiones, las provisiones, la sanidad y la información como parte de la cadena que abastece las unidades militares. Como parte de esta preparación en el arte de la guerra se considera que el triángulo logístico es un aspecto fundamental de la formación: la estrategia, la táctica y la logística.

\section{Contexto empresarial}

Los avances logrados por la logística en el ámbito militar han sido aprovechados por el ámbito empresarial, pues muchas de sus teorías y aplicaciones han tenido éxito en el desarrollo de las actividades propias de la organización empresarial para el logro de la eficacia y eficiencia que se le exige constantemente. Esto se presentó debido a que

El mundo empresarial comienza a darse cuenta del interés de los enfoques y técnicas de la logística cuando, a lo largo del período expansivo que sigue al fin de la Segunda Guerra Mundial, cambian de forma notable las condiciones del entorno y, como consecuencia, los enfoques de gestión tradicionales se revelan como relativamente ineficaces (Carrasco Arias, 2000, p. 19).

Esta etapa de transformaciones alude al periodo comprendido entre 1945 y 1973, en el que ocurre la "crisis del petróleo" y el crecimiento sostenido de la demanda (con tasas por encima del $10 \%$ anual), entre otros aspectos. De esta forma, tales cambios constantes en el contexto empresarial llegaron a incluir temas como el servicio al cliente, la innovación, la competencia en el tiempo, la producción masiva, etc., que han estado generando desafíos de sostenibilidad y competitividad para todas las organizaciones. 
Así, el problema de movilización de bienes que se ha planteado el ser humano en la guerra o en la empresa aún sigue vigente; no obstante, en lo que concierne al ámbito empresarial, solo hasta después de 1950 los gerentes se empezaron a preocupar por desarrollar este proceso en las organizaciones de una manera más confiable y con menores costos (García Olivares, 2004).

De acuerdo con esta preocupación, la logística empezó a hacer su aporte para aumentar la eficiencia en el almacenamiento y movimiento de bienes y servicios, así como de la información generada, con el fin de brindarle satisfacción al consumidor a un costo razonable (Ballou, 1999); todo ello en función de contribuir al logro de la estabilidad y el desarrollo de las organizaciones.

Por ello se afirma ahora que los empresarios del siglo XXI deben poseer conocimientos generales y específicos sobre la gestión de la cadena de suministro (Supply Chain ManagementSCM-) que les permitan planificar, implementar y controlar estos procesos de almacenamiento y circulación de productos y de información, desde el punto de compra de los materiales hasta el punto de consumo final, siempre con el objeto de satisfacer las necesidades de los consumidores (Lambert et al., 1998).

Estos procesos de evolución que ha tenido la logística en el campo empresarial han sido mostrados muy bien por autores como Fernández Sánchez (1994) y pueden ser planteados en cinco etapas relacionadas con las diferentes décadas de la segunda mitad del siglo XX.

La primera etapa (década de 1950): en esta etapa se reconoció el potencial integrador de la logística y se incluyó en ella el manejo de los costos. Se identificó cierta dificultad para implementarla en las organizaciones debido a los diversos procesos que se desarrollaban en ellas (Fontanet, 2000), entre los cuales se encontraba el aspecto referido a las necesidades de los clientes. Esto llevó a que en esta década también se integrara a la logística y a la producción el concepto de servicio al cliente y la evaluación de su percepción.

La segunda etapa (década de 1960): en esta fase se empieza a incorporar el concepto de Outsourcing a la logística y sus operaciones, pues la implementación de la logística empezaba a verse como un tema que requería un alto nivel de especialización y toda una infraestructura para la operación. En ese momento se comienza a valorar tanto los conocimientos propios de la producción como aquellos referidos a los servicios multifuncionales, los cuales serían denominados posteriormente como Third Party Service Suppliers o 3PL (Third Party Logistics) (Hendrickson, 1997).

La tercera etapa (década de 1970): en este momento se renueva el interés por el desempeño operacional interno de una compañía, pues se empiezan a incorporar en las empresas los temas de calidad y de manejo de tiempo en la producción (Jayaraman, Guide, \& Srivastava, 1999), y se comienza a pensar en los procesos desde la fase de adquisición de materiales hasta la entrega en el punto de consumo. Esta etapa y sus desarrollos fueron influidos y apalancados por los desarrollos de las nuevas tecnologías de la información que se dieron en ese momento.

La cuarta etapa (década de 1980): en este periodo se empiezan a vincular con la logística aspectos relacionados con el cuidado del medioambiente, de forma que los procesos de las organizaciones mejoraran el entorno o redujeran sus impactos negativos en él. Esto surge de la fuerza mundial que toma aspectos referidos al manejo de residuos, al reciclaje, al ahorro de energía, al efecto invernadero y a la gestión ecológica, entre otros, que posteriormente generarían la noción de "logística inversa". 
A partir de 1990 se presenta la quinta etapa: en este periodo, más reciente, se integra de manera más formal el componente ecológico a la logística, hasta llegar a configurar realmente la noción y las prácticas de la logística inversa, entendida como "el proceso de planificación, ejecución y control del flujo eficiente y rentable de las materias primas, inventarios en proceso, productos terminados e información relacionada desde el punto de consumo hasta el punto de origen con el fin de recapturar su valor o disponer de ellos correctamente" ${ }^{2}$ (Rogers \& Tibben Lembke 1998, p. 262). Como consecuencia de ello se empieza a hablar en la logística de cuestiones como la economía de los recursos, el manejo y eliminación de desechos, la transformación productiva, la productividad con bajo impacto medioambiental, etc. Como parte de este proceso también se empieza a configurar el concepto de logística verde.

Por supuesto, este tema no ha quedado circunscrito exclusivamente al ámbito privado (empresas), sino que también ha empezado a implicar la participación del sector público. Esto se debe a la necesidad normativa que estos temas han adquirido, pues se afirma que llegan a afectar la sostenibilidad y la competitividad tanto de las empresas como de las ciudades y naciones.

\section{La logística en el contexto colombiano}

Los procesos de internacionalización que se han generado en Colombia recientemente han producido cambios significativos en las condiciones de operación del país y de sus organizaciones, al punto que exigió transformar la visión tradicional de la logística (como una cuestión exclusiva de las empresas del sector privado) para avanzar hacia una perspectiva más contemporánea (que involucra el desarrollo económico, social y ecológico del país).

\section{Contexto militar colombiano}

En cuanto al desarrollo histórico de la logística en Colombia, el sistema logístico del Ejército se constituye en un caso de estudio bastante interesante y que cuenta con gran reconocimiento en el campo. Dicho reconocimiento se deriva del desarrollo y de la complejidad logística que ha implicado el apoyar a toda la estructura del Ejército durante varias décadas, teniendo en cuenta las características de guerra irregular que se ha tenido como parte del conflicto nacional y las difíciles condiciones geográficas y climáticas del país en las cuales opera.

La logística colombiana tiene su origen en la visión del general Francisco de Paula Santander y que fue la que apalancó los triunfos del ejército patriota en la batalla de Boyacá el 7 de agosto de 1819. Desde entonces la logística ha sido un apoyo esencial para el desarrollo de las demás armas del Ejército (Ejército Nacional de Colombia, 2012). Así, las Fuerzas Militares colombianas han logrado constituir uno de los más eficientes cuerpos logísticos para las labores de planeación, adquisición, distribución y administración de recursos que sustentan las operaciones militares (Gonzalez Muñoz, 1991).

\footnotetext{
2 Traducción de los autores.
} 
Con esta complejidad en mente, sumado a las consideraciones de carácter legal, aquellos miembros que forma parte de esta arma del Ejército (logística) deben tener un amplio conocimiento en materia presupuestal, legislativa (Ley 80 de contratación estatal) y normativa (normas de comercio internacional). Lo anterior se justifica en el hecho de que la logística militar colombiana, en su proceso de planeación, adquisición, producción y distribución, al igual que en todas las demás entidades del Estado, está regida por el sistema legal de contratación que ha establecido el país, y también en el hecho de que el material y equipo de guerra en el exterior requiere de diligencia en la realización de los trámites de importación y nacionalización.

Asimismo, el personal logístico del país debe estar preparado para almacenar el material comprado o producido para las diferentes unidades militares, teniendo presente que este material de intendencia, armamento y provisiones debe ser almacenado en óptimas condiciones de conservación y de seguridad, dadas sus características; para ello el Ejército cuenta con bodegas especialmente diseñadas para este fin. Posteriormente, este material debe ser distribuido a tiempo y en óptimas condiciones en cada una de las unidades militares, incluyendo aquellas ubicadas en lugares de difícil acceso.

Asimismo, se destaca que esta fuerza posee sus propias plantas para confeccionar uniformes, botas de combate, artículos de madera de uso privativo, raciones de campaña, municiones, armas y equipos, así como para la repotenciación de motores y para el mantenimiento de equipos de comunicación y optrónicos ${ }^{3}$, entre otros.

De esta forma, el sistema logístico del Ejército colombiano cumple entonces con las tareas de intendencia, armamento y equipo; transportes; comunicaciones; instalaciones; sanidad; mantenimiento; manejo de presupuesto y de adquisiciones, y, en forma general, con la administración de todos los recursos.

Finalmente, entendiendo que la logística apoya las operaciones militares en tiempos de guerra, pero también en tiempos de paz, es posible que esta disciplina pueda apoyar en la atención de las necesidades de la nación en el marco que propone un entorno de posconflicto y posacuerdo en aspectos como los siguientes: mejorando las capacidades para la seguridad nacional, cuidando las fronteras, manteniendo la seguridad ciudadana, atendiendo desastres, apoyando la construcción de infraestructuras para la consolidación de la paz, protegiendo el medio ambiente, atendiendo las áreas de concentración, ocupando el territorio, generando presencia del Estado, entre otras áreas estratégicas, tanto para la nación como para la implementación de los acuerdos. Es por ello que el sistema logístico del Ejército y de las Fuerzas Militares colombianas se encuentra en proceso constante de reestructuración.

\section{Contexto gubernamental colombiano}

En Colombia, el tema de la logística ha estado adquiriendo gran protagonismo, hasta el punto que ha sido incluido en los planes nacionales de desarrollo de los últimos gobiernos. Este es el caso del plan 2010-2014, que en su capítulo dos ("Convergencia y desarrollo regional, caracterización, dinámica y desafíos”) (Departamento Nacional de Planeación, 2011, p. 25) y su capítulo tres ("Crecimiento sostenible y competitividad") (Ibid., p. 61) hace referencia al tema.

3 Se entiende como sistema optrónico un conjunto de "elementos como radar, láser y calculadores electrónicos generalmente aplicado a la detección y seguimiento automático de blancos” (Real Academia de Ingeniería - RAING, s. f.). 
Asimismo, también en el plan nacional de desarrollo 2014-2018 la logística mantiene gran importancia para el gobierno nacional, pues forma parte de las estrategias transversales (Departamento Nacional de Planeación, 2015), a saber:

- Competitividad e infraestructura estratégicas (p. 107)

- Movilidad social (p. 265)

- Transformación del campo (p. 415)

- Crecimiento verde (p. 653)

- Seguridad, justicia y democracia para la construcción de paz (p. 447)

- Buen gobierno (p. 569), entre otros aspectos.

Es claro entonces que la infraestructura y competitividad estratégicas son necesarias para fomentar el crecimiento, el desarrollo humano y la integración y conectividad entre los territorios y la nación. Para lograrlo, en los últimos años el país ha hecho progresos importantes en materia de infraestructura y competitividad, y va a seguir haciendo esfuerzos con el ambicioso programa de infraestructura de transporte de Cuarta Generación (4G). No obstante, aún hoy se sigue contando con una porción importante de infraestructura obsoleta y sin mantenimiento, al punto que el país se está quedando rezagado frente al resto del continente.

Además, para desarrollar la competitividad del país en la actualidad ya no solo se requiere invertir en la construcción de carreteras, sino también trabajar en acciones que permitan la articulación del sistema de ciencia, tecnología e innovación $(\mathrm{C}+\mathrm{T}+\mathrm{i})$ con las organizaciones, así como una mayor eficiencia en el sistema judicial y un mejoramiento de las condiciones del capital humano vinculado a la logística. En este sentido, la estrategia referida a infraestructura y competitividad es clave para que Colombia pueda articularse con la economía mundial y disfrutar de mejores condiciones para la sostenibilidad.

Por su parte, la política nacional de competitividad y productividad de Colombia (Consejo Nacional de Política Económica y Social - Conpes, 2008a) destaca la logística como un valioso componente para alcanzar los objetivos estratégicos de la nación, mientras que la política nacional logística (Consejo Nacional de Política Económica y Social - Conpes, 2008b) muestra la necesidad de avanzar en aspectos importantes para el país en materia logística, tales como la generación de información en logística, el desarrollo de corredores logísticos articulados, la promoción del comercio exterior, la promoción del uso de las TIC como apoyo a la logística, el fomento y la provisión y la diversificación de los servicios de calidad en logística y transporte, entre otros. Todo ello se establece con el fin de optimizar el funcionamiento del sistema logístico nacional y de generar una reducción de los costos logísticos en Colombia, de forma que se pueda mejorar la eficiencia en toda la cadena logística del comercio exterior.

Adicionalmente, a través de la Mesa Sectorial de Logística ${ }^{4}$ el país ha estado realizando estudios periódicos en relación con la situación, condiciones y tendencias de la logística colombiana. El organismo ha realizado varias publicaciones que ha divulgado con el nombre de Caracterización

4 La Mesa Sectorial de Logística es un órgano de concertación voluntaria entre el gobierno, el sector productivo y la academia. En ella participan el Ministerio del Trabajo, el Ministerio de Educación, el Ministerio de Comercio y el SENA, institución que ha asumido su liderazgo. 
de la Logistica en Colombia (1999, 2006 y 2014). En su última edición (Fundación Centro de Investigaciones y Asistencia Internacional John F. Kennedy -CIATI JFK-, 2014), esta publicación analizó el desempeńo logístico de las organizaciones de Colombia en las dimensiones económica, organizacional, tecnológica, ocupacional, educativa y ambiental, y mostró las tendencias del sector de la logística en el país.

Este estudio muestra que todas las empresas, sin importar su tamańo, tienen un alto potencial de mejoramiento en cuanto se refiere a la organización logística, exceptuando el caso de las microempresas, que aún puede presentar dificultades. Allí se indica también la necesidad de actualizar en el país los programas de capacitación y formación del talento humano que se desempeńa en la logística, para adecuarlos a las macrotendencias globales en el campo.

En cuanto al tema de formación del talento humano en el campo de la logística, se destacan los siguientes aspectos:

- La mayoría de empresas no tienen programas formales de capacitación, formación y entrenamiento en logística (por competencias) sobre las tecnologías que utilizan, y poco incorporan profesionales con especialización, maestría y doctorado.

- En el nivel estratégico y táctico de las organizaciones logísticas, la mayor parte del talento humano tiene formación de pregrado (45\% y $32 \%$, respectivamente). Ya en el nivel operacional, la mayor parte de las personas poseen este tipo de formación (85\%). Esto afecta la productividad y competitividad del sector logístico. La falta de dominio del inglés es otro aspecto relevante.

- La vinculación de talento humano en el sector logístico, por región, es la siguiente: Centro: 93,44 \% tiene vinculados profesionales, 39,34 \% especialistas, 8,20 \% magísteres, 36,07 \% técnicos laborales, 31,15\% técnicos profesionales y 45,90 \% tecnólogos. Norte: $92,31 \%$ tiene vinculados profesionales, 46,15\% especialistas, 15,38 \% magísteres, $15,38 \%$ técnicos laborales, $61,54 \%$ técnicos profesionales y 23,98 \% tecnólogos. Occidente: $75 \%$ tiene vinculado profesionales, $50 \%$ especialistas, 6,25\% magísteres, $12,50 \%$ técnicos laborales, 37,50\% técnicos profesionales y 68,75\% tecnólogos. Oriente: $84,62 \%$ tiene vinculados profesionales, 38,46\% técnicos laborales, $15,38 \%$ técnicos profesionales y no tiene especialistas, magísteres ni tecnólogos.

- Los colectivos con mayor necesidad de formación son la región Occidente (Cali y Buenaventura), y la región Centro (Bogotá D.C., Medellín y Armenia).

- Las necesidades de formación de las empresas están concentradas en las áreas ocupacionales de "gestión logística", "almacenamiento", "transporte", "inventarios y materiales" y "servicio al cliente".

- Las empresas expresan la necesidad de capacitación en el idioma inglés.

- El nivel ocupacional donde se necesita urgentemente capacitación y entrenamiento es el "operacional", puesto que en este las ocupaciones han tenido una transformación más acelerada.

- Los altos costos logísticos vienen muchas veces más de la falta de capacitación, entrenamiento y formación de alta calidad, que de las deficiencias en infraestructura. 
- A pesar de ello, las empresas se encuentran más preocupadas por las diferentes tecnologías que por el talento humano dedicado a la logística (ejemplo de ello son la remuneración salarial, las condiciones contractuales de algunas empresas y los niveles de capacitación, entrenamiento y formación).

- Se necesita revisar el sistema de competencias laborales actuales, referidas a la logística, a la luz de las macrotendencias globales y las nuevas concepciones humanísticas relativas al desarrollo humano y la gestión del recurso humano.

- Es necesario hacer un monitoreo permanente sobre las macrotendencias globales que sirva de base para actualizar los programas de capacitación, entrenamiento y formación logística (en todos los niveles).

- Se requiere la creación de programas de pregrado en logística.

- Se requiere efectuar una revisión a los programas académicos de formación (IES) y en todos los niveles de capacitación y entrenamiento logístico para hacerlos más pertinentes y ajustarlos a los requerimientos de la Supply Chains Management y de la logística, así como a las macrotendencias globales, reemplazando los procedimientos empíricos que se usan actualmente.

- Se recomienda evitar confundir la Supply Chains Management y la logística con temas que pueden ser cercanos a campos como la ingeniería industrial, la administración de empresas, el marketing, entre otras.

- Se sugiere que en la educación en el campo se desarrollen estrategias pedagógicas que generen conciencia ambiental para el desarrollo de los procesos de la gestión logística.

- Debe trabajarse en el fortalecimiento del cuerpo profesoral de los programas logísticos con la incorporación de profesionales en temas de Supply Chains Management y logística.

Ahora bien, en el país también se cuenta con el Perfil Logístico de Colombia, cuya última versión se realizó para el año 2016 (Legiscomex, 2016). En este documento se evidencia el interés y desarrollo que el tema logístico ha tenido en Colombia y que ha generado un importante nivel de desarrollo, que se puede ver expresado en el desarrollo que han manifestado el tráfico portuario, el tráfico aéreo y el tráfico por carretera. Esta información se sistematiza en las tablas 1, 2 y 3.

Tabla 1. Evolución del tráfico portuario colombiano en toneladas (2013-2015)

\begin{tabular}{lrrr}
\hline \multicolumn{1}{c}{ Tráfico portuario } & \multicolumn{1}{c}{$\mathbf{2 0 1 3}$} & \multicolumn{1}{c}{$\mathbf{2 0 1 4}$} & \multicolumn{1}{c}{$\mathbf{2 0 1 5}$} \\
\hline Exportación & 134.143 .312 & 133.957 .730 & 127.976 .635 \\
Importación & 31.497 .870 & 34.152 .025 & 33.227 .186 \\
Cabotaje & 853.016 & 595.986 & 669.918 \\
Fluvial & 41.399 & 18.065 & 381.511 \\
Movilizaciones a bordo & 914 & 79.264 & 115 \\
\hline
\end{tabular}

Continúa tabla... 


\begin{tabular}{lrrr}
\hline \multicolumn{1}{c}{ Tráfico portuario } & \multicolumn{1}{c}{$\mathbf{2 0 1 3}$} & \multicolumn{1}{c}{$\mathbf{2 0 1 4}$} & \multicolumn{1}{c}{$\mathbf{2 0 1 5}$} \\
\hline No definido & 11.779 & 300 & 0 \\
Transbordo & 520.713 & 418.419 & 1.169 .702 \\
Tránsito internacional & 15.976 .292 & 18.343 .439 & 20.899 .002 \\
Transitoria & 136.711 & 131.018 & 120.869 \\
Productos (en blanco) & 15.716 & 571.679 & 14.191 .163 \\
\hline Total & 183.199 .735 & 188.269 .349 & 198.638 .116 \\
\hline
\end{tabular}

Fuente: Tomado de (Legiscomex, 2016, p. 10)

En la tabla 1 es evidente el desarrollo del tráfico portuario global entre 2013 y 2015, e incluso al menos desde el 2011 (Legiscomex, 2014). Esto también puede implicar un desarrollo importante del transporte marítimo en función del uso y condiciones de los ocho puertos existentes: Costa atlántica (Puerto Bolívar, Santa Marta, Barranquilla, Cartagena, Coveñas y Turbo) y Costa Pacífica (Buenaventura y Tumaco).

De la misma forma, en la tabla 2 se observa el desarrollo y comportamiento del transporte aéreo de carga en Colombia en los últimos años.

Tabla 2. Comportamiento del transporte aéreo de carga en toneladas (2014 - 2015)

\begin{tabular}{lccc|ccccccc}
\hline & \multicolumn{3}{c|}{ Nacional } & \multicolumn{5}{c}{ Internacional } \\
\cline { 2 - 9 } Periodo & \multirow{3}{*}{$\begin{array}{c}\text { Ro } \\
\text { Regular }\end{array}$} & Tegular & Total & \multicolumn{5}{c}{ Regular } & \multicolumn{4}{c}{ No Regular } \\
\cline { 5 - 10 } & & & Salida & Llegada & Total & Salida & Llegada & Total \\
\hline Ene.-Dic. 2014 & 148.145 & 14.263 & 162.678 & 328.763 & 195.278 & 524.014 & 38.813 & 18.142 & 56.955 \\
Ene.-Dic. 2015 & 162.854 & 16.494 & 179.347 & 324.747 & 191.963 & 516.710 & 52.962 & 20.778 & 73.739 \\
\hline
\end{tabular}

Fuente: Tomado de (Legiscomex, 2016, p. 43)

En este sentido, en cuanto al transporte aéreo, se puede destacar la existencia en el país de un buen número de aeropuertos con manejo de carga internacional, a saber: El Dorado en Bogotá; José María Córdova en Rionegro; Alfonso Bonilla Aragón en Palmira; Ernesto Cortissoz en Soledad; Rafael Núñez en Cartagena; Matecaña en Pereira; Palonegro en Lebrija; Camilo Daza en Cúcuta, y Gustavo Rojas Pinilla en San Andrés. De esta forma, Colombia cuenta con nueve puertos aéreos de estas características.

Asimismo, en la tabla 3 se detalla el desarrollo y comportamiento del transporte terrestre (por carretera) en Colombia en el ańo 2015. 
Tabla 3. Comportamiento del transporte por carretera en toneladas (aduanas de embarque de importación y exportación) (2015)

\begin{tabular}{lrrrrr}
\hline \multicolumn{2}{c}{$\begin{array}{c}\text { Aduanas de salida de las exportaciones } \\
\text { colombianas vía terrestre, 2015 }\end{array}$} & \multicolumn{2}{c}{$\begin{array}{c}\text { Aduanas de ingreso de las importaciones } \\
\text { colombianas vía terrestre, 2015 }\end{array}$} \\
\hline \multicolumn{1}{c}{ Aduana } & Toneladas & Participación & \multicolumn{1}{c}{ Aduana } & Toneladas & Participación \\
\hline Cúcuta & 761.600 & $47,5 \%$ & Cartagena & 1.737 .130 & $33,8 \%$ \\
Ipiales & 403.386 & $25,2 \%$ & Barranquilla & 545.186 & $27,7 \%$ \\
Maicao & 159.368 & $9,9 \%$ & Ipiales & 518.779 & $19,3 \%$ \\
Barranquilla & 153.446 & $9,6 \%$ & Bogotá & 446.023 & $11,9 \%$ \\
Cartagena & 63.051 & $3,9 \%$ & Cali & 111.762 & $3,3 \%$ \\
Santa Marta & 29.672 & $1,9 \%$ & Cúcuta & 72.469 & $1,0 \%$ \\
Cali & 23.042 & $1,4 \%$ & Medellín & 67.127 & $1,0 \%$ \\
Bogotá & 3.560 & $0,2 \%$ & Maicao & 64.932 & $0,6 \%$ \\
Puerto Asís & 2.967 & $0,2 \%$ & Puerto Asís & 40.735 & $0,6 \%$ \\
Pereira & 1.185 & $0,1 \%$ & Armenia & 25.154 & $0,3 \%$ \\
Buenaventura & 1.022 & $0,1 \%$ & Arauca & 16.723 & $0,2 \%$ \\
Medellín & 65 & $0,004 \%$ & Santa Marta & 14.761 & $0,168 \%$ \\
Arauca & 21 & $0,001 \%$ & Riohacha & 4.442 & $0,110 \%$ \\
Bucaramanga & 0.8 & $0,00005 \%$ & Pereira & 4.209 & $0,09552 \%$ \\
Leticia & 0.1 & $0,00001 \%$ & Bucaramanga & 2.771 & $0,04361 \%$ \\
Subtotal & 1.602 .386 & $100 \%$ & Subtotal & 3.672 .203 & $99,997 \%$ \\
Otros & 0 & $0 \%$ & Otros & 1.432 & $0.003 \%$ \\
\hline Total Exportaciones & $\mathbf{1 . 6 0 2 . 3 8 3}$ & $\mathbf{1 0 0} \%$ & Total Importaciones & $\mathbf{3 . 6 7 3 . 6 3 5}$ & $\mathbf{1 0 0 \%}$ \\
\hline & & & & & \\
\hline
\end{tabular}

Fuente: Tomado de (Legiscomex, 2016, pp. 38, 41)

Finalmente, en cuanto al tráfico terrestre se puede decir que el transporte de carreteras de Colombia se sustenta en una infraestructura vial conformada por 203.392 kilómetros. De este total, $27.577 \mathrm{~km}$ están a cargo de la nación, y de ellos (de los que han sido evaluados) solo 6.327,9 $\mathrm{km}$ se encuentran pavimentados, mientras que $2.425,7 \mathrm{~km}$ están sin pavimentar (de los 8.829,9 $\mathrm{km}$ de red primaria que han sido evaluados) (Legiscomex, 2016).

Por tanto, el tema de la logística en el país ha sido impulsado significativamente como consecuencia de las políticas de fomento al comercio exterior, las cuales han implicado los procesos de internacionalización económica y de globalización de todas las esferas de lo humano (homo- 
geneización). Esto se puede ver en la dinámica que han adquirido las relaciones comerciales de Colombia con otros países.

Un ejemplo de ello son los TLC de Colombia con Estados Unidos (aprobado por el Congreso de los Estados Unidos en el 2011), con Canadá (vigente desde agosto de 2011), con la Unión Europea (aprobado en 2012), con Chile (vigente a partir de 2009), con Corea del Sur (firmado en 2013), con México y con todos los países de América del Sur (menos las Guyanas), con la Comunidad Andina - CAN_ (componente comercial entre Bolivia, Colombia, Ecuador, Perú y Venezuela), con el Mercosur (que incorpora a Argentina, Brasil, Paraguay y Uruguay y a los países miembros de la CAN) y con Corea del Sur (Productos de Colombia, s. f.).

Por supuesto, esta dinámica internacional ha afectado la balanza comercial del país y ha incidido en el desarrollo de la logística. En la figura 2 se puede ver el comportamiento creciente de la balanza comercial (a valores constantes en millones de dólares FOB), el cual evidencia el aumento significativo que venía sufriendo el país, tanto en las importaciones como en las exportaciones, en los ańos recientes (datos a noviembre de 2016).

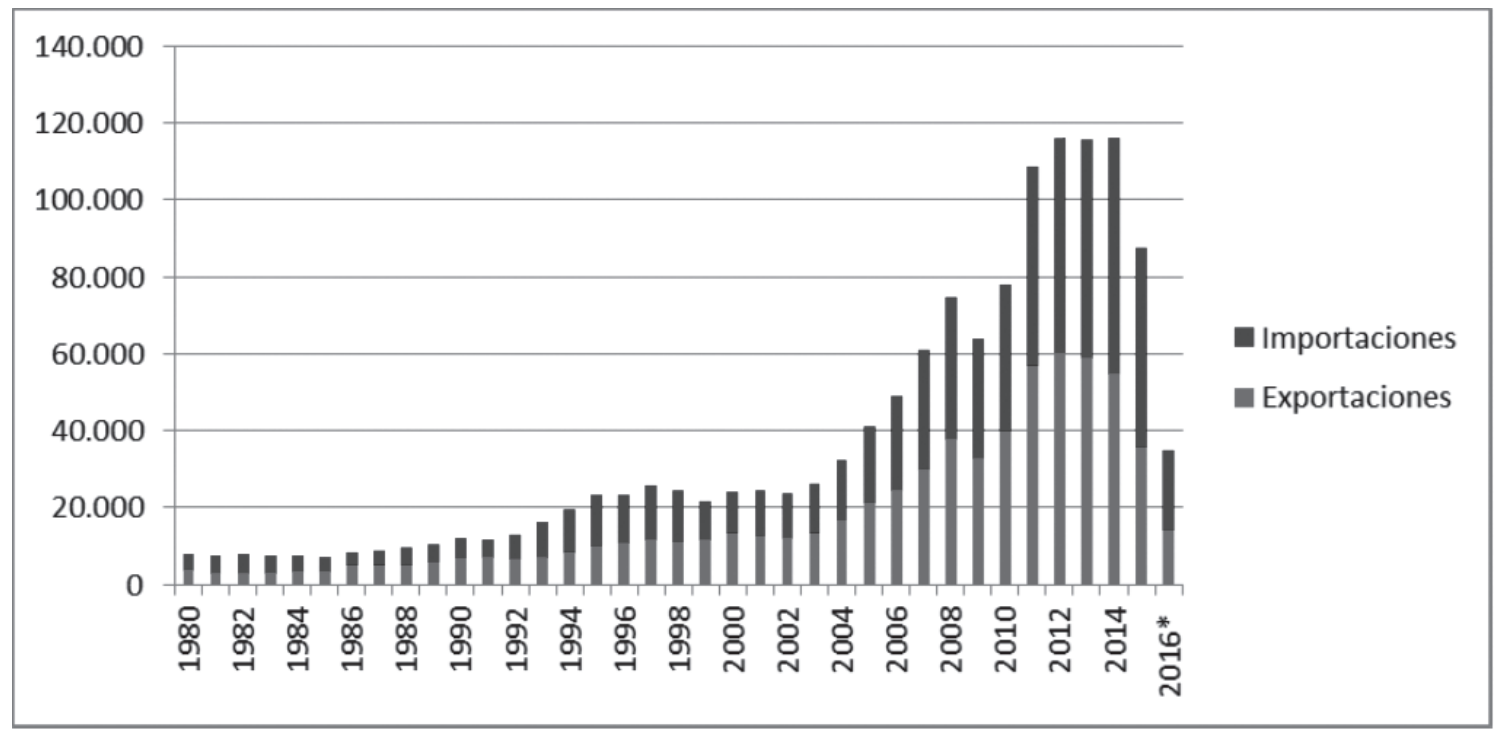

Figura 2. Balanza comercial de Colombia 1980-2016

Fuente: Elaborado por los autores con base en las cifras publicadas por el Departamento Administrativo Nacional de Estadística de Colombia -DANE- (2016)

En el 2013 el modo de transporte que predominaba en las exportaciones colombianas era el marítimo (con el $56 \%$ ), seguido por el terrestre (37\%) y el aéreo (7\%) Por su parte, el modo de transporte que predominaba para las importaciones era el modo marítimo (con el $73 \%$ ), el aéreo (22\%) y el terrestres (5\%) (Legiscomex, 2014). 


\section{Contexto empresarial colombiano}

En la actualidad, el panorama empresarial y de negocios para Colombia parece bastante positivo, puesto que, según el informe Doing Business ${ }^{5}$ en el 2016, el país ocupa el puesto 54 (tabla 4).

Tabla 4. Puesto de Colombia en la clasificación del Doing Business

\begin{tabular}{lc}
\hline Ańo & Puesto \\
\hline $\mathbf{2 0 1 6}$ & 54 \\
$\mathbf{2 0 1 5}$ & 52 \\
$\mathbf{2 0 1 4}$ & 43 \\
$\mathbf{2 0 1 3}$ & 45 \\
$\mathbf{2 0 1 2}$ & 42 \\
$\mathbf{2 0 1 1}$ & 39 \\
$\mathbf{2 0 1 0}$ & 37 \\
$\mathbf{2 0 0 9}$ & 53 \\
$\mathbf{2 0 0 8}$ & 66 \\
$\mathbf{2 0 0 7}$ & 79 \\
$\mathbf{2 0 0 6}$ & 76 \\
\hline
\end{tabular}

Fuente: (Banco Mundial \& Corporación Financiera Internacional, 2006, 2007, 2008, 2009, 2010, 2011, 2012, 2013, 2014, 2015, 2016).

Asimismo, según el Índice de Desempeño Logístico ${ }^{6}$, obtenido de la evaluación correspondiente al año $2014^{7}$ (Banco Mundial, 2014), Colombia se encuentra en el puesto 64 entre 160 países en relación con la percepción sobre su situación logística. En esta medición el país obtuvo una puntuación de 2.64, mientras que en el 2012 había obtenido 2.87 de puntaje y en 2010 una calificación de 2.77. Esto muestra que en los últimos tres años el país ha sido constante en relación con los criterios establecidos. Al verificar las diferentes variables que fueron valoradas en el campo logístico colombiano, se ven la infraestructura y el seguimiento y rastreo como aspectos por mejorar, pues en el primero el país ocupaba el lugar 98 en el mundo y en el segundo el 111 (El Tiempo, 2014a).

5 El Doing Business es una clasificación global hecha desde el 2004 que muestra la facilidad para hacer negocios en varios países del mundo (189 en el informe de 2015). Esto lo hace basándose en cinco criterios: eficiencia del proceso del despacho de aduana, calidad de infraestructura para el comercio y transporte, facilidad de acordar embarques a buenos precios, calidad de los servicios logísticos, capacidad para hacer seguimiento y rastrear los envíos y frecuencia de entregas de embarques oportunos.

6 El Índice de Desempeño Logístico es publicado también por el Banco Mundial como resultado de una evaluación que hace cada dos años acerca de la percepción que se tiene en relación con la logística de un país. En la medición la escala usada es de 1 a 5.

7 Última medición a la fecha (diciembre de 2016). 
Además, en el ranking establecido por el Índice Global de Competitividad ${ }^{8}$ 2014-2015 (Schwab, 2014), Colombia ocupa el puesto 66 y evidencia que ha tenido mejoras en materia de tecnología (en especial en cuanto a información y telecomunicaciones), aunque aún se presentan aspectos bastante problemáticos para poder avanzar en la clasificación: corrupción (primer lugar), infraestructura (segundo lugar) e ineficiencia burocrática (tercer lugar) (El Tiempo, 2014b).

Es por ello que Gómez Villamizar (2007) ya había afirmado que Colombia debe desarrollar la logística como elemento clave para lograr la competitividad en las empresas, puesto que sin un buen sistema logístico se hace muy difícil conseguir que los productos que se mueven a través del país no se vuelvan más costosos y, por ende, menos competitivos. Este autor opinaba que, en general, Latinoamérica se encuentra rezagada si se le compara con Norteamérica, Europa y algunos mercados del Pacífico. De la misma forma, Gómez consideraba que el costo logístico en Latinoamérica era muy alto (18 \% a $25 \%$ de costo logístico sobre el valor FOB de la mercancía), mientras que en Europa, Asia y Estados Unidos solo es del 10 al $12 \%$.

El análisis de este autor sobre el sistema logístico en Colombia muestra el inconveniente que representa, por un lado, que los centros de extracción primaria estén retirados de los centros de producción y, por el otro, que los centros de operación logística se encuentren retirados de los centros de consumo. Asimismo, se sabe que los sistemas férreos, fluviales y terrestres de Colombia no son los mejores, y por ello se hace necesario que el gobierno nacional continúe haciendo grandes inversiones en materia de infraestructura.

En cuanto a la logística inversa en Colombia, es posible afirmar que en el país se encuentran enormes deficiencias en su desarrollo sobre actividades, tales como el desensamblado para el desarrollo de nuevos productos (según las posibilidades que genera la modularidad), la inexistencia de centros especializados, la carencia de rutas de transporte para la recolección y la dificultad de acopio de los productos que están en desuso (provenientes de consumidores finales o intermedios) (Guerra, 2009).

Por último, según la Encuesta Nacional Logística (ENL) ${ }^{9}$ (Departamento Nacional de Planeación de Colombia, 2015), se puede afirmar que el costo logístico en el país es alto y que este se está incrementando (14,9\%) — como porcentaje de las ventas de productos de las empresas—, si se tiene en cuenta que está por encima del promedio de América Latina (14,7\%). Esto muchas veces se da como consecuencia de los excesivos tiempos en los procesos de transporte y esperas.

En dicha encuesta se encuentra también que los mayores costos de este campo en el país están en el transporte (37\%), almacenamiento (20\%); compras y manejo de proveedores (17 $\%)$; procesamiento de pedidos (10\%); planeación y reposición de inventarios (9\%) y logística

8 El Índice Global de Competitividad es un indicador desarrollado por el Foro Económico Mundial, que forma parte del Reporte Global de Competitividad (desde 2008), y que muestra el nivel de competitividad en varios países del mundo (144 en el informe de 2015). Los criterios usados son la productividad y la prosperidad generada con fundamento en 12 aspectos, incluidos aquellos referidos a instituciones, infraestructura, salud y educación, eficiencia del mercado laboral, preparación tecnológica, innovación y sofisticación de negocios.

9 La Encuesta Nacional Logística es una iniciativa del Departamento Nacional de Planeación (DNP) que se aplica a 768 empresarios (prestadores y usuarios de servicios logísticos), con el fin de analizar el desempeńo logístico de Colombia y de las organizaciones frente a las actuales exigencias del mercado, para articular el diseño y las políticas públicas en función de mejorar la productividad y competitividad de este. En ella los temas fundamentales están referidos al perfil empresarial, las perspectivas e innovación, el comercio exterior, las regiones y canales de distribución, el desempeńo y organización logística, la infraestructura y la tecnología, la educación y el empleo, entre otros. 
de reversa $(7 \%)$. También informa que las mayores dificultades se encuentran en los costos de transporte y en la infraestructura de puertos y aeropuertos, la falta de sistemas de información logística y de talento humano capacitado, la complejidad de los trámites de aduana y el precio de las bodegas.

La encuesta también muestra que un porcentaje importante (casi el $70 \%$ ) de empresarios consideran que la normatividad aduanera no genera suficiente seguridad para la realización de operaciones de comercio exterior ${ }_{s}$ que el nivel de innovación en las empresas logísticas es bajo (lo cual genera poco uso de operadores por parte de estas, apenas el 37,5\%), que las grandes empresas tercerizan más sus servicios logísticos (casi en el $50 \%$ ) y que, por el contrario, la pequeńa y mediana empresa no acuden a esta vía frecuentemente (solo 27,6 \% y 35,5\%, respectivamente).

Otro de los hallazgos de interés de la ENL es que solo el 92,2\% de las entregas se hacen completas, que solo el $80 \%$ de estas llegan a tiempo y que solo el 77,3 \% de las entregas cumplen los dos criterios. Asimismo, encontró que únicamente el 54,7 \% de las empresas cuentan con sistemas tecnológicos que permitan realizar la trazabilidad y el monitoreo de la carga.

En términos de la calificación general del tema logístico, que es la referencia para el país, el promedio nacional está en 5,45 sobre 10 . Con este referente, en lo que respecta a la logística, la región mejor evaluada es el Caribe $(5,98)$, seguida por el Centro-oriente $(5,73)$, el Eje cafetero y Antioquia $(5,66)$, y el Pacífico $(5,65)$. Por el contrario, las regiones peor evaluadas son el Centro-sur y Amazonia (5,09), y los Llanos orientales $(4,56)$, pues están por debajo del promedio nacional.

Estas evaluaciones muestran entonces aquellos aspectos fuertes y aquellas debilidades que cada una de las regiones presentaron. Según la ENL, en el caso del Caribe la fortaleza está en los servicios logísticos e industria $(6,45)$ y la debilidad está en logística urbana $(5,44)$; en el caso de Centro-oriente, la fortaleza está en infraestructura $(6,32)$ y la debilidad en la movilidad en las ciudades y el manejo de las restricciones a la movilidad de carga (4,94); en el caso del Eje cafetero y Antioquia, la fortaleza está en infraestructura $(6,33)$ y la debilidad en la consecución de cargas de retorno y gestión de contenedores; para el Pacífico, la fortaleza es en servicios logísticos e industria $(6,05)$ y la debilidad en la infraestructura, que no facilita las actividades de logística $(5,73)$; en los Llanos la fortaleza es la infraestructura $(5,13)$ y la debilidad es la oferta de servicios logísticos $(3,84)$. Finalmente, para el caso de la región Centro-sur Amazonia la fortaleza es la infraestructura $(5,53)$, mientras que las debilidades son la carga de retorno y la seguridad, pues no facilitan las actividades logísticas.

Si se tienen en cuenta los resultados generales y los análisis por regiones, se evidencia que a nivel país se debe trabajar en la mejora de aspectos referidos a: a) comercio exterior: agilizar y flexibilizar los trámites de aduanas; mejorar la atención de las entidades relacionadas con el comercio exterior - 7×24-; entender y cumplir la normatividad; agilizar la implementación del estatuto aduanero; mejorar la infraestructura; fortalecer la tecnología y el personal de los puertos; aumentar la eficiencia en las plataformas e infraestructura logística, y aumentar la inversión en infraestructura del transporte; $b$ ) desempeño y operación logística: ver la logística como área estratégica; aumentar la visibilidad y conectividad entre las cadenas de valor; aumentar la inversión en tecnología e infraestructura; desarrollar, innovar y especializar en valor agregado a los operadores logísticos del país, e implementar el cargue y descargue en las noches; $c$ ) transporte: aumentar la 
formalización del sector; lograr la interoperabilidad de los sistemas de información de transporte; mejorar la formación del sector transportador; promocionar e implementar el multimodalismo en el país; avanzar hacia la bancarización del sector transportador, e implementar las tecnologías para seguimiento y trazabilidad de la carga en el país; d) innovación: lograr la apropiación tecnológica para el proceso logístico; promocionar la investigación en redes logísticas; diseñar estrategias para el mejoramiento de la logística de última milla; desarrollar proyectos de logística sostenible y tejer redes colaborativas entre instituciones, regiones, empresas y sectores; y $e$ ) educación y empleo: fortalecer las competencias logísticas en el talento humano; ampliar la oferta académica en el sector logístico y aumentar la práctica en los programas académicos de logística.

Esta encuesta nacional se realizó en todas las regiones del país con la siguiente distribución geográfica: Caribe (11,7 \%), Eje cafetero y Antioquia (27,7 \%), Centro-oriente (49,3\%), Pacífico (7,2 \%), Llanos (1,4\%), Sur y Amazonia (2,6 \%). Además, esta encuesta muestra que, respecto a su nivel de desarrollo, el 26,9\% de los usuarios de la logística están en el nivel más básico, el $42,7 \%$ en el medio y el 30,5 \% en el avanzado. Por su parte, el 32,6 \% de los proveedores de estos servicios se encuentran en el nivel básico, el $40 \%$ en medio y el 27,4 \% en el avanzado.

Así, toda la dinámica del campo logístico ya descrito, tanto en el contexto militar como empresarial, se muestra muy favorable y en constante crecimiento. No obstante, este mismo hecho ha establecido nuevos retos frente al desarrollo del sector y requerimientos en múltiples campos, incluido el campo educativo en relación con los programas formativos. Por lo tanto, se hace evidente la necesidad de caracterizar la oferta educativa existente, de forma que se pueda mejorar la calidad de los programas, ofrecer alternativas frente a los existentes y, de ser el caso, ampliar y mejorar la cobertura en la formación de expertos sobre el tema.

\section{La formación en el campo logístico en Colombia}

Ya se ha mostrado en otros análisis la importancia de la formación en logística, incluso en relación con la necesidad de trabajar en ella a partir del enfoque educativo de formación por competencias (Sanabria Rangel \& Ospina Díaz, 2017).

No obstante, si se tiene en cuenta que muchos de los estudios sobre logística en el país han mostrado que uno de los aspectos relevantes para que este campo se desarrolle está relacionado con la formación en el campo logístico, el aumento de personal especializado en el tema, la ampliación de la oferta educativa, el mejoramiento de los programas existentes y la formación práctica en dichos programas, entonces parece necesario también empezar a preocuparse por caracterizar y hacer seguimiento a la oferta educativa que existe en el país en relación con el tema.

En este aparte se pretende sistematizar un análisis sobre el estado actual de la formación en el campo de la logística en Colombia. En función de este propósito se realizó un análisis del mercado educativo para efectuar un comparativo de la oferta actual en el ámbito señalado. Los resultados del análisis se sistematizan a continuación.

En la tabla 5 se muestran los programas de logística activos existentes en el país en función de la oferta por regiones y por modalidad. En ella se puede ver el nivel de desarrollo e interés que 
ha generado el campo de estudio en las diferentes zonas del país y en función de las modalidades en las que los programas se pueden desarrollar.

Tabla 5. Programas activos de logística en Colombia por departamento y por modalidad

\begin{tabular}{|c|c|c|c|c|}
\hline \multirow{2}{*}{$\begin{array}{l}\text { Departamento de oferta } \\
\text { del programa }\end{array}$} & \multicolumn{3}{|c|}{ Metodología } & \multirow[b]{2}{*}{ TOTAL } \\
\hline & $\begin{array}{c}\text { Distancia } \\
\text { (Tradicional) }\end{array}$ & Presencial & Virtual & \\
\hline Bogotá, D.C. & 3 & 38 & 4 & 45 \\
\hline Antioquia & 1 & 24 & 4 & 29 \\
\hline Valle del Cauca & & 19 & 2 & 21 \\
\hline Atlántico & & 18 & 2 & 20 \\
\hline Bolívar & 4 & 13 & 1 & 18 \\
\hline Santander & & 13 & 5 & 18 \\
\hline Cundinamarca & & 6 & & 6 \\
\hline Norte de Santander & & 3 & 2 & 5 \\
\hline Magdalena & & 5 & & 5 \\
\hline Risaralda & & 5 & & 5 \\
\hline Tolima & & 4 & & 4 \\
\hline Caldas & 2 & 1 & & 3 \\
\hline San Andrés y Providencia & & 2 & & 2 \\
\hline Meta & & 1 & & 1 \\
\hline Huila & & 1 & & 1 \\
\hline Cesar & & 1 & & 1 \\
\hline Nariño & & 1 & & 1 \\
\hline Quindío & & 1 & & 1 \\
\hline Casanare & & 1 & & 1 \\
\hline Cauca & & & & 0 \\
\hline Córdoba & & & & 0 \\
\hline Total & 10 & 157 & 20 & 187 \\
\hline
\end{tabular}

Fuente: (Ministerio de Educación Nacional - República de Colombia, 2015)

Se puede resaltar entonces que en la actualidad la formación logística está concentrada en los programas presenciales, con el $84 \%$, mientras que los programas a distancia solo representan el $16 \%$ (5\% tradicionales y $11 \%$ virtuales).

Asimismo, más del $20 \%$ de los programas existentes (en todas las modalidades) se encuentran concentrados en Bogotá, y más del 50 \% están concentrados entre Bogotá, Antioquia y Valle del Cauca. Por el contrario, las zonas del país en donde estos programas tienen un menor número son Meta, Cauca, San Andrés y Providencia, Huila, Cesar, Córdoba, Nariño, Quindío y 
Casanare, que sumados representan solo el $4 \%$ del total de programas existentes. Se resalta que Cundinamarca, siendo una zona central, solo representa el $3 \%$ de los programas, siendo todos presenciales.

Un análisis similar se puede plantear en relación con los programas existentes, aunque desde la perspectiva de los diferentes niveles de formación académica que se puede dar en la logística. En la tabla 6 se presentan los datos referidos al número de programas existentes en el país con respecto al criterio de niveles formativos.

Tabla 6. Programas activos de logística en Colombia por departamento y por nivel de formación

\begin{tabular}{|c|c|c|c|c|c|c|c|}
\hline \multirow{2}{*}{$\begin{array}{l}\text { Departamento } \\
\text { de oferta } \\
\text { del programa }\end{array}$} & \multicolumn{6}{|c|}{ Nivel de formación } & \multirow[b]{2}{*}{ Tota } \\
\hline & Doctorado & Maestría & Especialización & Universitaria & Tecnológica & $\begin{array}{l}\text { Form. } \\
\text { técnica }\end{array}$ & \\
\hline Bogotá, D.C. & & 3 & 14 & 9 & 14 & 5 & 45 \\
\hline Antioquia & & 3 & 13 & 1 & 10 & 2 & 29 \\
\hline Valle del Cauca & & 1 & 7 & 1 & 11 & 1 & 21 \\
\hline Atlántico & & 1 & 5 & & 12 & 2 & 20 \\
\hline Bolívar & & 2 & 6 & & 6 & 4 & 18 \\
\hline Santander & & & 5 & 1 & 6 & 6 & 18 \\
\hline Tolima & & & & & 2 & 2 & 4 \\
\hline Cundinamarca & 1 & & 1 & 2 & 2 & & 6 \\
\hline Norte de Santander & & & 3 & & 1 & 1 & 5 \\
\hline Magdalena & & & 4 & & & 1 & 5 \\
\hline Risaralda & & & 3 & & & 2 & 5 \\
\hline Caldas & & & & & 2 & 1 & 3 \\
\hline Meta & & & & 1 & & & 1 \\
\hline Cauca & & & & & & & 0 \\
\hline $\begin{array}{l}\text { San Andrés y } \\
\text { Providencia }\end{array}$ & & & & & & 2 & 2 \\
\hline Huila & & & & & & 1 & 1 \\
\hline Cesar & & & 1 & & & & 1 \\
\hline Córdoba & & & & & & & 0 \\
\hline Nariño & & & 1 & & & & 1 \\
\hline Quindío & & & 1 & & & & 1 \\
\hline Casanare & & & & & 1 & & 1 \\
\hline Total & 1 & 10 & 64 & 15 & 67 & 30 & 187 \\
\hline
\end{tabular}

Fuente: (Ministerio de Educación Nacional - República de Colombia, 2015) 
$\mathrm{Al}$ analizar la distribución de los programas por nivel de formación, se evidencia que la mayoría de programas en el campo logístico se encuentran concentrados en el nivel de especialización (posgrado) y en los programas tecnológicos (pregrado), de tal manera que entre los dos llegan al $70 \%$; esto contrasta con el hecho de que los programas de formación profesional son prácticamente el menor porcentaje (8\%). Otro aspecto interesante es que más del 50\% de la formación logística se encuentra más desarrollada en el nivel de formación técnica y tecnológica que en los niveles superiores.

Desde otro punto de vista, dentro del nivel tecnológico, la mayoría de programas se encuentran también en Bogotá, Antioquia, Valle del Cauca y Atlántico (61\% dentro de estos programas y $21 \%$ del total). Esto mismo ocurre en los programas tecnológicos, aunque en este caso la concentración es aún mayor (70 \% dentro de estos programas y $25 \%$ del total). No obstante, la situación cambia cuando se analiza lo que sucede con los programas de formación técnica profesional, pues los programas de este tipo se concentran en Bogotá, Bolívar y Santander (50 \% dentro de estos programas y $8 \%$ del total).

Adicionalmente, con base en los datos existentes también es posible determinar el nivel de interés que ha generado el tema de la logística en las instituciones educativas en relación con el sector al que pertenecen. Estos datos se muestran en la tabla 7.

Tabla 7. Programas de logística activos en Colombia por carácter académico y por sector

\begin{tabular}{|c|c|c|c|c|}
\hline \multirow{2}{*}{ Carácter académico de la institución } & \multirow{2}{*}{ Reconocimiento del Ministerio } & \multicolumn{2}{|c|}{ Sector } & \multirow{2}{*}{ Total } \\
\hline & & Oficial & Privada & \\
\hline Institución técnica profesional & Registro calificado & 3 & 5 & 8 \\
\hline \multicolumn{2}{|c|}{ Totales de instituciones técnicas profesionales } & 3 & 5 & 8 \\
\hline \multirow{2}{*}{ Institución tecnológica } & N/A & & 2 & 2 \\
\hline & Registro calificado & 10 & 15 & 25 \\
\hline \multicolumn{2}{|l|}{ Totales de instituciones tecnológicas } & 10 & 17 & 27 \\
\hline \multirow{3}{*}{$\begin{array}{l}\text { Institución universitaria/ } \\
\text { escuela tecnológica }\end{array}$} & N/A & & 1 & 1 \\
\hline & Registro alta calidad & 1 & & 1 \\
\hline & Registro calificado & 14 & 41 & 55 \\
\hline \multicolumn{2}{|c|}{ Totales de instituciones universitarias/escuelas tecnológicas } & 15 & 42 & 57 \\
\hline \multirow{2}{*}{ Universidad } & N/A & & 3 & 3 \\
\hline & Registro calificado & 26 & 66 & 92 \\
\hline \multicolumn{2}{|l|}{ Totales universidades } & 26 & 69 & 95 \\
\hline \multicolumn{2}{|l|}{ Total } & 54 & 133 & 187 \\
\hline
\end{tabular}

Fuente: (Ministerio de Educación Nacional - República de Colombia, 2015) 
En función de la información presentada se puede afirmar que la mayoría de los programas se encuentran concentrados en las organizaciones clasificadas como universidades (51\%) y como instituciones universitarias o escuelas tecnológicas (30\%).

Asimismo, el campo logístico parece haber generado mayor interés en las instituciones educativas de carácter privado, pues allí es donde se presenta la mayor concentración de programas (71 \%), con mayor claridad también en las universidades e instituciones universitarias. Se resalta el hecho de que entre todos los programas existentes solo uno de ellos se encuentra acreditado, correspondiente a una institución universitaria del sector público.

Por último, en la tabla 8 se sistematiza la información relacionada con los programas del campo logístico de acuerdo con su clasificación en función de las diferentes áreas de conocimiento, en los niveles de pregrado y posgrado.

Tabla 8. Programas académicos activos de logística en Colombia según su clasificación por área de conocimiento y por nivel de formación

\begin{tabular}{|c|c|c|c|c|}
\hline \multirow{2}{*}{ Área de conocimiento } & \multirow{2}{*}{ Nivel de formación } & \multicolumn{2}{|c|}{ Nivel académico } & \multirow{2}{*}{ Total } \\
\hline & & Posgrado & Pregrado & \\
\hline \multirow{2}{*}{ Bellas artes } & Formación técnica profesional & & 1 & 1 \\
\hline & Tecnológica & & 1 & 1 \\
\hline \multicolumn{2}{|l|}{ Totales bellas artes } & & 2 & 2 \\
\hline \multirow{5}{*}{$\begin{array}{l}\text { Economía, administra- } \\
\text { ción contaduría y afines }\end{array}$} & Especialización & 30 & & 30 \\
\hline & Formación técnica profesional & & 27 & 27 \\
\hline & Maestría & 5 & & 5 \\
\hline & Tecnológica & & 47 & 47 \\
\hline & Universitaria & & 15 & 15 \\
\hline \multicolumn{2}{|c|}{ Totales economía, administración, contaduría y afines } & 35 & 89 & 124 \\
\hline \multirow{5}{*}{$\begin{array}{l}\text { Ingeniería, arquitectu- } \\
\text { ra, urbanismo y afines }\end{array}$} & Doctorado & 1 & & 1 \\
\hline & Especialización & 34 & & 34 \\
\hline & Formación técnica profesional & & 2 & 2 \\
\hline & Maestría & 5 & & 5 \\
\hline & Tecnológica & & 19 & 19 \\
\hline \multicolumn{2}{|c|}{ Totales ingeniería, arquitectura, urbanismo y afines } & 40 & 21 & 61 \\
\hline \multicolumn{2}{|l|}{ Total } & 75 & 112 & 187 \\
\hline
\end{tabular}

Fuente: (Ministerio de Educación Nacional - República de Colombia, 2015) 
Una vez analizadas las diferentes áreas de conocimiento en que se circunscriben los programas en logística se encontró que la mayoría de ellos están caracterizados como programas del área económica, administrativa y afines $(66 \%)$, mientras que un porcentaje importante se encuentra ubicado como programas del área de ingeniería, arquitectura y afines. El 1 \% de los programas se caracterizan como correspondientes al área de las bellas artes.

En la primera área de clasificación, la mayoría de programas se encuentran ubicados en el nivel tecnológico (38 \% dentro de la categoría), mientras que en la segunda área de clasificación la mayoría de los programas pertenecen al nivel de especialización (56\%).

En cuanto al nivel de formación, en las tres áreas se encuentra un mayor número de programas en el nivel de pregrado que de posgrado ( $72 \%$ en el área económica, administrativa y afines, y $34 \%$ en el área de ingeniería, arquitectura y afines). Con esto se tiene que, en general, los programas de pregrado son mayoritarios frente a los de posgrado (60\% entre todos los programas).

\section{Comentarios finales}

Aunque la logística ha estado presente a lo largo de la historia, desde el ámbito militar, en la época actual se ha convertido en un aspecto fundamental para las organizaciones y las naciones (dado el modelo e imaginario económico vigente) en función de su estabilidad y desarrollo; por esto se considera que este se constituye en un concepto y una práctica de carácter estratégicos.

Dado que la logística se encarga de mejorar los flujos de productos, servicios e información de las organizaciones en función de las necesidades sociales y ambientales, y enmarcado en criterios de eficacia, eficiencia y costo razonable, se trata entonces de un aspecto muy importante si se pretende lograr una auténtica sostenibilidad para la actividad social, económica y organizacional.

En este sentido, el tema se ha vuelto tan relevante que empieza a ser transversal a toda la actividad organizacional, a tal punto que ahora guarda relación con la gestión general, la estrategia, los modelos integrados de gestión, la cadena de suministro, la sostenibilidad, la Responsabilidad Social Empresarial, la gestión ambiental, el valor compartido, la gestión de calidad, la gestión de producción, la gestión comercial, la gestión de la información, el servicio al cliente, el outsourcing, la cadena de valor, entre otros.

Esta importancia ha generado iniciativas internacionales para caracterizar al sector, tales como el Doing Business del Banco Mundial, el Índice de Desempeño Logístico y el Índice Global de Competitividad, que hacen seguimiento permanente a las condiciones de desarrollo logístico, no solo de las empresas y demás organizaciones, sino también de las diversas naciones.

En el caso de Colombia, la logística ha estado adquiriendo gran importancia y reconocimiento por sus logros, devenidos también del campo militar. Esto se ha dado de tal forma que en el país este tema ya no corresponde solo al ámbito de las empresas y demás organizaciones, sino que ha captado el interés y participación del Estado mismo, sobre todo si se consideran los aspectos de la discusión previa.

En respuesta a este interés, en Colombia se han creado lineamientos, estrategias, políticas, leyes y normas en relación con el campo logístico que le permitan desarrollar sus ventajas compe- 
titivas y generar sostenibilidad en el tiempo, sobre todo en una dinámica como la que plantea el escenario del posconflicto.

Como consecuencia de ello, el país ha incorporado propósitos de carácter logístico en los planes nacionales de desarrollo y ha definido la política nacional de competitividad y productividad (Conpes, 2008a), la política nacional logística (Conpes, 2008b), la Mesa Sectorial de Logística y la Encuesta Nacional Logística. Esto ha generado también iniciativas como las referidas al Perfil Logístico de Colombia.

Aunque en Colombia las acciones referidas a infraestructura, el trabajo en la seguridad de las vías, los desarrollos en los marcos y seguridad jurídicos, el desarrollo del sistema de investigación, desarrollo e innovación ( $\mathrm{I}+\mathrm{D}+\mathrm{i})$ y la integración Universidad-Empresa-Estado (U-E-E), entre otros, se consideran importantes, un aspecto fundamental para acompańar estos esfuerzos en el fortalecimiento logístico es el mejoramiento de las condiciones y procesos formativos del capital humano vinculado al sector. Esto es tan trascendental que en el país se considera que la carencia o mala formación ocasiona costos logísticos más altos que la falta de infraestructura.

Este hecho se explicita en los diferentes estudios que se han realizado sobre el sector, como las caracterizaciones periódicas (1999, 2006 y 2014), en las cuales se muestra la necesidad de contar con programas formales de capacitación en las empresas, de niveles crecientes de formación académica en el talento humano, de crear programas de pregrado en logística, de aumentar la cantidad de trabajadores capacitados, de persistir en el bilingüismo (inglés), de trabajar en la formación por competencias logísticas, de aumentar la práctica en los programas académicos existentes, etc.

Como consecuencia de ello, en Colombia, según las estadísticas del SNIES a fecha de julio de 2015, existen 238 programas de logística, de los cuales únicamente están activos 187 . De este número de programas, 10 se desarrollan con metodología a distancia (tradicional), 157 con metodología presencial y 20 con metodología virtual.

Además, se observa que Bogotá concentra el mayor número de programas activos en el campo con 45, seguido de Antioquia con 29, Valle del Cauca con 21 y Atlántico con 20. A nivel nacional se encontró como oferta académica activa en el campo 1 programa de doctorado, 10 maestrías, 64 especializaciones, 30 programas técnicos profesionales, 67 tecnologías y 15 programas profesionales.

Asimismo, se puede ver que los programas de logística son ofrecidos en las áreas de conocimiento de bellas artes ( 2 programas, ambos de pregrado); economía, administración contaduría y afines (124 programas, 35 de posgrado y 89 de pregrado); y en ingeniería, arquitectura, urbanismo y afines (61 programas, 40 son de posgrado y 21 de pregrado).

Esto contrasta un poco con los lineamientos estratégicos que han estado definiendo los gobiernos para el país y que pretenden incorporar esfuerzos en materia logística en las diferentes regiones. Se afirma esto porque las acciones que se han emprendido no han estado acompańadas por un crecimiento, desarrollo, actualización y mejoramiento sólido de los programas de formación en ellas. De hecho, si se observa la actual situación de los programas académicos de logística en dichas regiones, no parece existir homogeneidad en su desarrollo ni correspondencia con estos esfuerzos y acciones regionales. 
Un ejemplo de ello es que mientras la región Centro es la que más vinculación hace de profesionales en el sector logístico $(93,44 \%)$, en ella solo el $11 \%$ de los programas son de nivel universitario o profesional; adicionalmente, esta región cuenta con un 8,20 \% de magísteres, frente al $5 \%$ de programas de este nivel en la región; tiene el 39,34\% de especialistas, en comparación con el $34 \%$ que constituyen los programas de especialización en el campo en la región; el 45,90 $\%$ de tecnólogos contrasta con el $32 \%$ de oferta formativa en este nivel en la región, y vincula el $31,15 \%$ de técnicos profesionales, cuando solo el $17 \%$ de los programas de la región están en este nivel. Esto representa unos porcentajes de $7 \%, 3 \%, 21 \%, 20 \%$ y $11 \%$ del total nacional, respectivamente.

De igual forma, en el caso de la región Norte contrastan los resultados del nivel de formación que existe en las personas contratadas con el número de programas existentes para cada nivel: 92,31\% de profesionales vinculados frente al $0 \%$ de oferta en ese nivel; 15,38\% de magísteres frente al $7 \%$ de oferta; 46,15\% de especialistas frente a $35 \%$ de oferta; 23,98 \% tecnólogos frente al $39 \%$ de oferta; y 61,54\% técnicos profesionales frente al $20 \%$ de oferta.

Por su parte, la situación de la región Occidente es la siguiente: $75 \%$ de profesionales vinculados en las organizaciones con respecto a una oferta de $5 \%$ en ese nivel; 6,25\% de magísteres con respecto a una oferta de $5 \%$ en ese nivel; $50 \%$ de especialistas con respecto a una oferta de $36 \%$ en ese nivel; 68,75\% de tecnólogos con respecto a una oferta de $50 \%$ en ese nivel; 37,50 $\%$ de técnicos profesionales con respecto a una oferta de $5 \%$ en ese nivel.

Finalmente, para el caso de la región Oriente, las organizaciones manifestaron tener vinculado el 84,62 \% de su personal en el nivel profesional, en relación con una oferta de los programas de la región en ese nivel de 50 \%; 0 \% de magísteres vinculados en relación con una oferta de $0 \%$ en ese nivel; $0 \%$ de especialistas en relación con una oferta de $0 \%$ en ese nivel; $0 \%$ de tecnólogos en relación con una oferta de $50 \%$ en ese nivel; $15,38 \%$ de técnicos profesionales en relación con una oferta de $0 \%$ en ese nivel.

Asimismo, parecen ser acertadas las apreciaciones de los empresarios frente a la necesidad de contar con programas de formación en las regiones, pues, por ejemplo, en la región Occidente (Cali y Buenaventura, principalmente) se percibe carencia de programas, y al consultar la oferta se encuentra que la región Pacífico solo cuenta con el $12 \%$ del total de programas en todos los niveles de formación. En este sentido, las regiones parecen constituirse en una buena oportunidad para ampliar la oferta formativa y contribuir a la formación de los expertos que dichas regiones requieren, en todos los niveles de formación.

Llama la atención también el hecho de que este comentario se manifiesta de igual forma en la región Centro (Bogotá D.C., Medellín y Armenia, principalmente), siendo que es allí donde se presenta la mayor concentración de programas académicos en el campo (63\% del total nacional en los distintos niveles de formación). Esto puede mostrar que, a pesar del crecimiento de la oferta, la región ha tenido un crecimiento aún mayor en el campo logístico que demanda un número cada vez mayor de trabajadores expertos. Si esto es cierto, parece constituirse también en un campo interesante de desarrollo profesional para quienes se desempeñan en él.

En cuanto a la expectativa de los empresarios frente a la necesidad de fortalecer el nivel de capacitación, sobre todo en el nivel operacional, se debe mencionar que esto implicaría para la academia ampliar el número y ajustar el perfil de los programas para acomodarlos a la significa- 
tiva transformación que se ha dado en ese nivel. Al parecer esto cuenta con el acompañamiento del sector educativo, pues al revisar el total de programas en los niveles de formación que corresponden al nivel operacional, se encuentra que en la oferta en el nivel tecnológico los programas representan el $36 \%$ del total nacional, y en el caso del nivel técnico profesional, el $16 \%$ del total nacional (52\% respecto al total de programas existentes en el país).

Asimismo, cuando se observa la solicitud de los empresarios sobre la necesidad de crear más programas de pregrado en logística, se ve el potencial de crecimiento de este tipo de programas en el país, pues se alude al hecho de que 112 programas (de 187) son insuficientes para el desarrollo que ha tenido el sector y para las oportunidades y retos que se plantean hacia el futuro.

Finalmente, se puede afirmar que toda la dinámica que se ha generado en el campo de la logística, tanto a nivel militar como empresarial y académica, ha sido muy favorable, pues las cifras muestran que Colombia es una plaza con gran dinamismo en el campo logístico. Asimismo, ha generado gran movimiento en cuanto a programas formativos, puesto que, dada la actual situación de nuestro país, en el campo logístico se requieren cada vez más profesionales con conocimientos especializados en logística.

Por supuesto, las recomendaciones efectuadas en los distintos estudios sobre el tema de la logística apuntan a la necesidad de avanzar también cualitativamente en la caracterización de estos programas, puesto que la sola existencia de mayor número de estos y mayor diversidad (con el consecuente aumento en el número de egresados para el campo) no garantizan que los programas se ajusten a las necesidades del país y de las organizaciones, aunque, por supuesto, sí constituye un avance en la dirección requerida.

\section{Referencias}

1. Ballou, R. H. (1999). Business Logistics Management (4 ed.). Upper Saddle River, Nueva Jersey (Estados Unidos): Prentice-Hall International, Inc.

2. Ballou, R. H. (2004). Logística: Administración de la cadena de suministro (5 ed.). México: Pearson.

3. Banco Mundial. (2014). Indice de desempeño logístico. Recuperado de http://datos.bancomundial.org/indicador/ LP.LPI.OVRL.XQ

4. Banco Mundial \& Corporación Financiera Internacional. (2006). Doing Business 2006: Creando empleos (3 ed.). Washington D.C.: Banco Mundial.

5. Banco Mundial \& Corporación Financiera Internacional. (2007). Doing Business 2007: Cómo reformar (4 ed.). Washington D.C.: Banco Mundial.

6. Banco Mundial \& Corporación Financiera Internacional. (2008). Doing Business 2008: Comparando la regulación en 178 economias (5 ed.). Washington D.C.: Banco Mundial.
7. Banco Mundial \& Corporación Financiera Internacional. (2009). Doing Business 2009: Comparando la regulación en 181 economias (6 ed.). Washington D.C.: Banco Mundial.

8. Banco Mundial \& Corporación Financiera Internacional. (2010). Doing Business 2010: Reformar en tiempos dificiles (7 ed.). Washington D.C.: Banco Mundial.

9. Banco Mundial \& Corporación Financiera Internacional. (2011). Doing Business 2011: Creando oportunidades para los emprendedores (8 ed.). Washington D.C.: Banco Mundial.

10. Banco Mundial \& Corporación Financiera Internacional. (2012). Doing Business 2012: Haciendo negocios en un mundo más transparente (9 ed.). Washington D.C.: Banco Mundial.

11. Banco Mundial \& Corporación Financiera Internacional. (2013). Doing Business 2013: Regulaciones inteligentes para pequeñas y medianas empresas (10 ed.). Washington D.C.: Banco Mundial. 
12. Banco Mundial \& Corporación Financiera Internacional. (2014). Doing Business 2014: Entendiendo las regulaciones para las pequeñas y medianas empresas (11 ed.). Washington D.C.: Banco Mundial.

13. Banco Mundial \& Corporación Financiera Internacional. (2015). Doing Business 2015: Going Beyond Efficiency (12 ed.). Washington D.C.: Banco Mundial.

14. Banco Mundial \& Corporación Financiera Internacional. (2016). Doing Business 2016: Measuring Regulatory Quality and Efficiency (13 ed.). Washington D.C.: Banco Mundial.

15. Carrasco Arias, J. (2000). Evolución de los enfoques y conceptos de la logística: su impacto en la dirección y gestión de las organizaciones. Economía Industrial (331), 17-34.

16. Cedillo Campos, M. G., \& Sánchez Ramírez, C. (2008). Análisis dinámico de sistemas industriales. México: Trillas.

17. Cipoletta Tomassian, G., Pérez Salas, G., \& Sánchez, R. J. (2010). Politicas integradas de infraestructura, transporte y logistica: Experiencias internacionales y propuestas iniciales (Vol. 150). Santiago de Chile: Comisión Económica para América Latina y el Caribe (CEPAL).

18. Closs, D. J., \& Stank, T. P. (1999). A cross-functional curriculum for supply chain education at Michigan State University. Journal of Business logistics, 20(1), 59-72.

19. Consejo Nacional de Política Económica y Social - Conpes. (2008a). Documento Conpes 3527: Politica nacional de competitividad y productividad.

20. Consejo Nacional de Política Económica y Social Conpes. (2008b). Documento Conpes 3547: Politica nacional logistica.

21. Council of Supply Chain Management Professionals CSCMP. (2013). Supply Chain Management terms and glossary (pp. 222). Illinois: Council of Supply Chain Management Professionals - CSCMP.

22. Departamento Administrativo Nacional de Estadística de Colombia -DANE-. (2016, Junio de 2016). Comercio internacional - Balanza Comercial. Recuperado de http:// www.dane.gov.co/index.php/comercio-y-servicios/comercio-exterior/balanza-comercial

23. Departamento Nacional de Planeación - República de Colombia. (2011). Plan Nacional de Desarrollo 20102014: Prosperidad para todos. Bogotá D.C.: Departamento Nacional de Planeación - República de Colombia.

24. Departamento Nacional de Planeación - República de Colombia. (2015). Plan Nacional de Desarrollo 2014-2018: Todos por un nuevo país. Bogotá D.C.: Departamento Nacional de Planeación - República de Colombia.

25. Departamento Nacional de Planeación de Colombia. (2015). Colombia es Logística. Encuesta Nacional de Logística - Resultados nacionales 2015. Bogotá: Departamento Nacional de Planeación (Colombia).
26. Ejército Nacional de Colombia. (2012). Cuerpo Logístico y Administrativo. Recuperado de http://www.ejercito.mil. co/?idcategoria $=253837$

27. El Tiempo. (2014a, Mayo 19 de 2014). Colombia, puesto 97 en desempeńo logístico. El Tiempo. Recuperado de http:// www.eltiempo.com/economia/sectores/logistica-en-colombia/14007019

28. El Tiempo. (2014b, Septiembre 3 de 2014). Lo bueno y lo malo de Colombia para competir en el mundo. El Tiempo. Recuperado de http://www.eltiempo.com/economia/indicadores/colombia-en-el-ranking-de-competitividad-del-foro-economico-mundial/14475537

29. Fernández Sánchez, E. (1994). Dirección de la producción: Métodos operativos. Madrid: Civistas.

30. Fontanet, L. (2000). Gestión de residuos urbanos. Madrid: ExLibris.

31. Fundación Centro de Investigaciones y Asistencia Internacional John F. Kennedy -CIATI JFK-. (2014). Caracterización del sector de la logística en Colombia 2014. Bogotá: Servicio Nacional de Aprendizaje (SENA), Mesa Sectorial de Logística.

32. García Olivares, A. A. (2004). Recomendaciones táctico-operativas para implementar un programa de logistica inversa: Estudio de caso en la industria del reciclaje de plásticos (pp. 163). México: Eumednet.

33. Gómez Villamizar, F. J. (2007, Agosto 27 de 2007). Logística, el dolor de cabeza para la competitividad. Revista Dinero.

34. Gonzalez Muñoz, F. (1991, Junio 15 de 1991). La logística militar: Una empresa modelo. El Tiempo. Recuperado de http://www.eltiempo.com/archivo/documento/MAM103507

35. Guerra, H. S. (2009). Inventarios: Manejo y control. Bogotá: ECOE.

36. Hendrickson, C. C. (1997). Introduction to green design. Green Design Initiative, 329-405.

37. Jayaraman, V. G., Guide, V. D. R., \& Srivastava, R. (1999). A closed-loop logistics model for remanufacturing. Journal of Operational Research Society, 50(5), 497-508.

38. Klaus, E. (1999). Sistemic competitiveness economics. Instituto para la ciencia y la cooperación, 50-57.

39. Lambert, D. M., Cooper, M., \& Pagh, J. D. (1998). Supply Chain Management: Implementation issues and research opportunities. The International Journal of Logistics Management, 9(2), 1-19.

40. Legiscomex. (2014). Perfil logístico de Colombia 2014. Bogotá D.C.: Legiscomex.

41. Legiscomex. (2015). Logística -logistics (Glosario). Re-cuperado de http://www.legiscomexalinstante.com/GlosarioTermino2009. asp?CodIdioma=ESP\&CodGlosario $=1052$ 
42. Legiscomex. (2016). Perfil logístico de Colombia 2016 Bogotá D.C.: Legiscomex.

43. López Torres, V. G., Alcalá Álvarez, M. C., Plascencia López, I., \& Marín Vargas, M. E. (2010). Identificación de necesidades de formación de profesionales en las áreas de logística y la administración de la cadena de suministro. Análisis cualitativo en Baja California. Revista del Centro de Investigación, 9(33), 75-85.

44. Mendoza Torres, M. R. (2013). Rúbrica de evaluación de competencias profesionales para un curso de Logística, en programas de Ingeniería. Revista de Tecnología, 12(Esp), 57-67.

45. Ministerio de Educación Nacional - República de Colombia. (2015). Sistema Nacional de Información de la Educación Superior -SNIES-. Recuperado de http://snies. mineducacion.gov.co/consultasnies/programa

46. Narasimhan, S. L., McLeavey, D. W., \& Billington, P. J. (1996). Planeación de la producción y control de inventarios (2. ${ }^{\mathrm{a}}$ ed.). México: Prentice Hall Hispanoamericana.

47. Posada Zamudio, E. (1999). La logística militar y sus aplicaciones en la logística empresarial: Logística naval operacional. Pensamiento \& Gestión(6), 71-87.

48. Productos de Colombia. (s. f.). Con qué países tiene Colombia tratados de libre comercio? Recuperado de http://www.productosdecolombia.com/main/guia/TLC_ Paises_Libre_Comercio_Colombia.asp
49. Puentes Garzón, H. J. (2006). Caracterización de la logística en Colombia 2006 (2.a ed.). Bogotá: Servicio Nacional de Aprendizaje (SENA), Mesa Sectorial de Logística.

50. Real Academia de Ingeniería - RAING. (s. f.). Diccionario Español de Ingeniería. Madrid: Real Academia de Ingeniería.

51. Real Academia Española - RAE. (2001). Diccionario de la lengua española - DRAE (S.L.U. Espasa Libros Ed. 22. ${ }^{a}$ ed.). Madrid: S.L.U. Espasa Libros.

52. Rogers, D. S., \& Tibben Lembke, R. S. (1998). Going backwards: Reverse logistics trends and practices. Reno: Nevada University, Center for Logistics Management.

53. Sanabria Rangel, P.E., \& Ospina Díaz, M. R. (2017). Competencias profesionales en el campo de logistica para Latinoamérica: Un análisis desde Colombia. Documento pendiente de publicación.

54. Schmidheiny, S. (1992). The business logic of sustainable development. Columbia Journal of World Business, 27(3/4), 18-24.

55. Schwab, K. (2014). The global competitiveness report 20142015. Geneva (Switzerland): World Economic Forum.

56. Velásquez Contreras, A. (2003). Modelo de gestión de operaciones para PYMES innovadoras. Revista EAN (47), 66-87. 